\title{
Combinatorial interpretations of particular evaluations of complete and elementary symmetric functions
}

\author{
Pietro Mongelli \\ Dipartimento di Matematica "G. Castelnuovo" \\ Università "la Sapienza" di Roma \\ Rome, Italy \\ mongelli@mat.uniroma1.it
}

Submitted: Nov 30, 2011; Accepted: Mar 14, 2012; Published: Mar 31, 2012

Mathematics Subject Classifications: 05A19 (Primary); 05A05, 05A30, 11P81 (Secondary)

\begin{abstract}
The Jacobi-Stirling numbers and the Legendre-Stirling numbers of the first and second kind were first introduced by Everitt et al. (2002) and (2007) in the spectral theory. In this paper we note that Jacobi-Stirling numbers and Legendre-Stirling numbers are specializations of elementary and complete symmetric functions. We then study combinatorial interpretations of this specialization and obtain new combinatorial interpretations of the Jacobi-Stirling and Legendre-Stirling numbers.
\end{abstract}

Keywords: Jacobi-Stirling numbers, Legendre-Stirling numbers, symmetric functions, combinatorial interpretations.

\section{Introduction}

The aim of this paper is to give combinatorial interpretations of a family of numbers which includes the Legendre-Stirling numbers and Jacobi-Stirling numbers of both kinds. The Jacobi-Stirling numbers were introduced in [7] as the coefficients of the integral composite powers of the Jacobi differential operator

$$
\mathfrak{l}_{\alpha, \beta}[y](t)=\frac{1}{(1-t)^{\alpha}(1+t)^{\beta}}\left(-(1-t)^{\alpha+1}(1+t)^{\beta+1} y^{\prime}(t)\right)^{\prime}
$$

with fixed real parameters $\alpha, \beta>-1$. When the parameters are both equal to 0 , we find the definition of the Legendre-Stirling numbers, first introduced in [6] and later studied in $[1]$. In $[6,7,13]$ the authors show that both numbers share many properties with the classical Stirling numbers of both kinds such as similar recurrence relations, generating functions and total positivity properties. Recently several combinatorial interpretations 
of the Legendre-Stirling numbers $([2,5])$ and the Jacobi-Stirling numbers ([8]) have been given, most of which are based on permutations and partitions, exactly the same combinatorial objects used for the classical interpretations of the Stirling numbers.

In this paper we note that the Jacobi-Stirling numbers and the Legendre-Stirling numbers of both kinds are specializations of elementary and complete homogeneous symmetric functions. We study these specializations and then apply the results to Jacobi-Stirling and Legendre-Stirling numbers. More precisely, we give general combinatorial interpretations of these specializations with a unified approach. These combinatorial interpretations include new combinatorial interpretations for the Jacobi-Stirling and Legendre-Stirling numbers as well as the results in [2, Theorem 2], [5, Theorem 2.5] and [8, Theorem 7].

The organization of the paper is as follows. In Section 2 we recall the symmetric functions and some of their properties and show how we can obtain Jacobi-Stirling numbers and Legendre-Stirling numbers by a suitable evaluation of them. In Section 3 we define a $q$-analogue of these numbers and we give a combinatorial interpretations of them that generalizes interpretations given in $[2,5,8]$ when $q=1$ and that we can use in particular for the Jacobi-Stirling numbers. The evaluation of the symmetric functions that we use are parameterized by real nonnegative numbers. As done in [8] for the Jacobi-Stirling numbers, in Section 4 we study our evaluations of the symmetric functions as polynomials in these parameters. In Section 5 we turn our attention to other functions, already introduced in [3], that generalized the complete and elementary symmetric functions. We give a combinatorial interpretation of their evaluations and if we apply it to the case of Jacobi-Stirling numbers we get a new result. Moreover in this section we study the evaluations of the well known monomial symmetric functions. Finally, in Section 6 we recall other properties of the elementary and symmetric functions such that if applied to the Jacobi-Stirling numbers and Legendre-Stirling numbers gives us immediately some properties studied in $[1,6,7]$.

\section{Definitions, notation and preliminaries}

We let $\mathbb{P}:=\{1,2,3, \ldots\}, \mathbb{N}:=\mathbb{P} \cup\{0\}, \mathbb{Z}=\mathbb{N} \cup\{-1,-2,-3, \ldots\}$. The cardinality of a set $A$ will be denoted by $|A|$.

For the following definitions we use the notations of [12, Chapter I.2]. Consider the ring $\mathbb{Z}\left[x_{1}, \ldots, x_{n}\right]$ of polynomials in $n$ independent variables $x_{1}, \ldots, x_{n}$ with integer coefficients. For each $r \geq 0$ the $r$-th elementary symmetric function $e_{r}$ is the sum of all products of $r$ distinct variables $x_{i}$, so that $e_{0}=1$ and for $r \geq 1$

$$
e_{r}\left(x_{1}, \ldots, x_{n}\right)=\sum_{i_{1}<i_{2}<\cdots<i_{r}} x_{i_{1}} x_{i_{2}} \cdots x_{i_{r}}
$$

The $r$-th complete symmetric function $h_{r}$ is the sum of all monomials of total degree $r$ in the variables $x_{1}, \ldots, x_{n}$ so that $h_{0}=1$ and for $r \geq 1$

$$
h_{r}\left(x_{1}, \ldots, x_{n}\right)=\sum_{i_{1} \leq i_{2} \leq \cdots \leq i_{r}} x_{i_{1}} x_{i_{2}} \cdots x_{i_{r}}
$$

The Electronic Journal of COMBinatorics 19 (2012), \#P60 
It is a simple exercise to check the following recursion formulas for $n, j \geq 1, n>j$ :

$$
\begin{aligned}
h_{n-j}\left(x_{1}, \ldots, x_{j}\right) & =h_{n-j}\left(x_{1}, \ldots, x_{j-1}\right)+x_{j} h_{n-j-1}\left(x_{1}, \ldots, x_{j}\right) \\
e_{n-j}\left(x_{1}, \ldots, x_{n-1}\right) & =e_{n-j}\left(x_{1}, \ldots, x_{n-2}\right)+x_{n-1} e_{n-j-1}\left(x_{1}, \ldots, x_{n-2}\right)
\end{aligned}
$$

with initial conditions

$$
\begin{aligned}
& h_{0}\left(x_{1}, \ldots, x_{j}\right)=1 ; \quad h_{n-j}\left(x_{1}, \ldots, x_{j}\right)=0 \quad(\text { if } n<j), \\
& e_{0}\left(x_{1}, \ldots, x_{n-1}\right)=1 ; \quad e_{n-j}\left(x_{1}, \ldots, x_{n-1}\right)=0 \quad(\text { if } n<j) \text {. }
\end{aligned}
$$

We now recall the definitions of the Jacobi-Stirling numbers and the Legendre-Stirling numbers and some of their basic properties.

The Jacobi-Stirling numbers of the second kind $J S_{n}^{(j)}(z)$ are defined for all $n, j \in \mathbb{N}$ by Everitt et al. via the following expansion of the $n$-th composite power of $\mathfrak{l}_{\alpha, \beta}$ (see [7, Theorem 4.2]):

$$
(1-t)^{\alpha}(1+t)^{\beta} \mathfrak{l}_{\alpha, \beta}^{n}[y](t)=\sum_{j=0}^{n}(-1)^{j}\left(J S_{n}^{(j)}(\alpha+\beta+1)(1-t)^{\alpha+j}(1+t)^{\beta+j} y^{(j)}(t)\right)^{(k)}
$$

where $\mathfrak{l}_{\alpha, \beta}$ is the Jacobi differential operator (1) and $\alpha, \beta>-1$ are fixed real parameters. Since these numbers depend only on the sum $\alpha+\beta$, we set $z=\alpha+\beta+1>-1$.

In $[7$, Section 4] or in [8, Section 1] the following recursive formula are given

$$
J S_{n}^{(j)}(z)=J S_{n-1}^{(j-1)}(z)+j(j+z) J S_{n-1}^{(j)}(z)
$$

with initial conditions

$$
J S_{n}^{(0)}(z)=J S_{0}^{(j)}(z)=0 ; \quad J S_{0}^{(0)}(z)=1 .
$$

From [7, Theorem 4.1] relations (8), (9) can be easily deduced.

Moreover, in [7] the following equation is given:

$$
x^{n}=\sum_{j=0}^{n} J S_{n}^{(j)} \prod_{i=0}^{j-1}(x-i(z+i))
$$

and in the same paper the authors define the (unsigned) Jacobi-Stirling numbers of the first kind for all $n, j \in \mathbb{N}$ as follows

$$
\prod_{i=0}^{n-1}(x-i(z+i))=\sum_{j=0}^{n}(-1)^{j} J c_{n}^{(j)} x^{j}
$$

The Jacobi-Stirling numbers of the first kind satisfy the following recursion

$$
J c_{n}^{(j)}(z)=J c_{n-1}^{(j-1)}(z)+(n-1)(n-1+z) J c_{n-1}^{(j)}(z)
$$


with initial conditions

$$
J c_{n}^{(0)}(z)=J c_{0}^{(j)}(z)=0 ; \quad J c_{0}^{(0)}(z)=1 .
$$

It is simple to check that recursion (4) is the same as (8) if $x_{j}=j(j+z)$ for all $j \geq 1$ and that recursion (5) is the same as (12) if $x_{n-1}=(n-1)(n-1+z)$ for all $n \geq 2$.

The Legendre-Stirling numbers were first introduced in [6] as the coefficients of the integral powers of the second-order Legendre differential operator $\mathfrak{l}[\cdot]$ (1) when $\alpha=\beta=0$. Therefore these numbers coincide with the Jacobi-Stirling numbers when $z=1$. As noted in [6] and [7], both numbers have properties similar to the classical Stirling numbers of both kinds. This is because the Stirling numbers also satisfy the same recursion formulas (4) and (5) with $x_{j}=j$ for all $j \geq 1$. For these reasons we define the following objects.

Fix an integer $r \geq 1$ and fix $a_{1}, \ldots, a_{r}$ nonnegative real numbers. Let $f(x)=(x+$ $\left.a_{1}\right)\left(x+a_{2}\right) \cdots\left(x+a_{r}\right)$. We then define for all $n, j \in \mathbb{N}$

$$
\begin{aligned}
H_{j, n}^{f} & =h_{n-j}(f(1), f(2), \ldots, f(j)) ; \\
E_{j, n}^{f} & =e_{n-j}(f(1), f(2), \ldots, f(n-1)) .
\end{aligned}
$$

By the above remarks, and denoting with $S(n, j), c(n, j)$ the (unsigned) Stirling numbers of the second and first kind, and with $L S(n, j), L c(n, j)$ the (unsigned) LegendreStirling numbers of the second and first kind, it is easy to check that

$$
\begin{aligned}
S(n, j) & =H_{j, n}^{x} ; & c(n, j) & =E_{j, n}^{x} ; \\
L S_{n}^{(j)} & =H_{j, n}^{x(x+1)} ; & L c_{n}^{(j)} & =E_{j, n}^{x(x+1)} ; \\
J S_{n}^{(j)}(z) & =H_{j, n}^{x(x+z)} ; & J c_{n}^{(j)}(z) & =E_{j, n}^{x(x+z)} .
\end{aligned}
$$

When the polynomial $f$ is in $\mathbb{N}[x]$ it is possible to define a $q$-analogue of the numbers $H_{j, n}^{f}$ and $E_{j, n}^{f}$.

Given a positive integer $n$, we denote by $[n]$ the polynomial $1+q+\cdots+q^{n-1}$; moreover, we set $[0]:=0$.

Definition 1. Let $f(x)=\left(x+a_{1}\right) \cdots\left(x+a_{r}\right)$, with $r \in \mathbb{P}$ and $a_{1}, \ldots, a_{r} \in \mathbb{N}$. For all $j, n \geq 1$, we define the polynomials $H_{j, n}^{f}(q)$ by the recursive formula

$$
H_{j, n}^{f}(q)=H_{j-1, n-1}^{f}(q)+\left[j+a_{1}\right]\left[j+a_{2}\right] \cdots\left[j+a_{r}\right] H_{j, n-1}^{f}(q) ;
$$

with initial conditions

$$
H_{0, n}^{f}(q)=H_{j, 0}^{f}(q)=0 ; \quad H_{0,0}^{f}(q)=1 .
$$

For all $j, n \geq 1$, we define the polynomials $E_{j, n}^{f}(q)$ by the recursive formula

$$
E_{j, n}^{f}(q)=E_{j-1, n-1}^{f}(q)+\left[n-1+a_{1}\right]\left[n-1+a_{2}\right] \cdots\left[n-1+a_{r}\right] E_{j, n-1}^{f}(q) ;
$$

with initial conditions

$$
E_{0, n}^{f}(q)=E_{j, 0}^{f}(q)=0 ; \quad E_{0,0}^{f}(q)=1 .
$$


In the case of the Stirling numbers we recognize well-known $q$-analogue (see e. g. $[4,10,11])$.

In the following, for all $j, n, k \in \mathbb{N}$ we denote by $H_{j, n}^{f}[k]$ and $E_{j, n}^{f}[k]$ the coefficient of $q^{k}$ in $H_{j, n}^{f}(q)$ and $E_{j, n}^{f}(q)$, respectively.

In the next section we give a combinatorial interpretation for $H_{j, n}^{f}[k]$ and $E_{j, n}^{f}[k]$; in particular we obtain a combinatorial interpretation of $H_{j, n}^{f}$ and $E_{j, n}^{f}$. This combinatorial interpretation is new even for $f=x(x+z)$ (i. e., for the Jacobi-Stirling numbers). In Section 4 we consider $H_{j, n}^{f}$ and $E_{j, n}^{f}$ as polynomials in $a_{1}, \ldots, a_{r}$ where $f(x)=(x+$ $\left.a_{1}\right) \cdots\left(x+a_{r}\right)$ and we give other combinatorial interpretations.

\section{Combinatorial interpretations of $H_{j, n}^{f}[k]$ and $E_{j, n}^{f}[k]$}

In this section we give a combinatorial interpretation of the coefficients $H_{j, n}^{f}[k]$ and $E_{j, n}^{f}[k]$ for any fixed polynomial with only integer roots and nonnegative coefficients $f=(x+$ $\left.a_{1}\right)\left(x+a_{2}\right) \cdots\left(x+a_{r}\right), a_{1} \leq a_{2} \leq \cdots \leq a_{r}$, and for fixed $j, n, k \in \mathbb{N}$.

\subsection{Combinatorial interpretation of $H_{j, n}^{f}[k]$.}

Fix $j, n, k \in \mathbb{N}$ and consider $r$ labeled copies of the numbers $1, \ldots, n$, i. e.

$$
1_{1}, 1_{2}, \ldots, 1_{r}, 2_{1}, 2_{2}, \ldots, 2_{r}, \ldots \ldots, n_{1}, n_{2}, \ldots, n_{r} .
$$

We consider a pair $P=\left(\pi,\left(S_{1}, \ldots, S_{a_{r}}\right)\right)$ where $\pi$ is a set partition of a subset of $\left\{1_{1}, \ldots, n_{r}\right\}$ into $j$ blocks and $S_{1}, \ldots, S_{a_{r}}$ are subsets of $\left\{1_{1}, \ldots, n_{r}\right\}$. We say that $P$ is $f$-Stirling of order $(n, j)$ if $P$ is a partition of $(21)$ into $j+a_{r}$ subsets such that

- the subsets in $\pi$ are nonempty and each one contains the minimum number with all its indices;

- one of the subsets in $\pi$ contains $1_{1}, 1_{2}, \ldots, 1_{r}$;

- each $m_{i},(1 \leq m \leq n, 1 \leq i \leq r)$ is in one of the first $j+a_{i}$ subsets.

For example, an $f$-Stirling partition of order $(3,2)$, with $f=(x+1)(x+2)$, is $\left(\left\{1_{1}, 1_{2}, 3_{1}\right\},\left\{2_{1}, 2_{2}\right\},\{\},\left\{3_{2}\right\}\right)$. The partitions $P_{1}=\left(\left\{1_{1}, 1_{2}, 2_{1}\right\},\left\{2_{2}, 3_{1}, 3_{2}\right\},\{\},\{\}\right)$, $P_{2}=\left(\left\{2_{1}, 2_{2}\right\},\left\{3_{1}, 3_{2}\right\},\left\{1_{1}, 1_{2}\right\},\{\}\right)$ and $P_{3}=\left(\left\{1_{1}, 1_{2}, 3_{2}\right\},\left\{2_{1}, 2_{2}\right\},\{\},\left\{3_{1}\right\}\right)$ are not $f$ Stirling of order $(3,2)$ since one of the previous conditions fails.

Now, given an $f$-Stirling partition $P=\left(\pi,\left(S_{1}, \ldots, S_{a_{r}}\right)\right)$ of order $(n, j)$, we label each subset in $P$ in the following way: each one of the subsets in $\pi$ is labeled by the minimum number that it contains; each $S_{i}$ is labeled by $1-i$ for all $i=1, \ldots, a_{r}$. For example, the sets in $\left(\left\{1_{1}, 1_{2}, 3_{1}\right\},\left\{2_{1}, 2_{2}\right\},\{\},\left\{3_{2}\right\}\right)$ have labels $(1,2,0,-1)$. With these labels, we can define a total order relation between the subsets of $P$. Moreover we say that a subset is greater or less than a number via its label. By using these order relations we can define the following numbers. 
Let $i_{j}(i \leq n, j \leq r)$ be a labeled number in (21) and let $A_{i_{j}}$ be the unique subset in $P$ containing $i_{j}$. We set

$$
s_{i_{j}}:=\mid\left\{A \text { subset in } P \mid A_{i_{j}}<A<i\right\} \mid
$$

and

$$
s_{P}:=\sum_{1 \leq i \leq n, 1 \leq j \leq r} s_{i_{j}}
$$

We define $s_{P}:=0$ when $n=0$. For example, if $P=\left(\left\{1_{1}, 1_{2}, 3_{1}\right\},\left\{2_{1}, 2_{2}\right\},\{\},\left\{3_{2}\right\}\right)$ then $s_{3_{1}}=1, s_{3_{2}}=3$ and all other $s_{i_{j}}$ are 0 , so $s_{P}=4$.

We then have the following result.

Proposition 2. For all $n, j, k \in \mathbb{N}$ we have that $H_{j, n}^{f}[k]$ is the number of $f$-Stirling partitions $P$ of order $(n, j)$ such that $s_{P}=k$.

Proof. For all $n, j \geq 0$ we consider the following generating function

$$
p_{j, n}^{f}(q):=\sum_{P} q^{s_{P}}
$$

where the sum is over all $f$-Stirling partitions $P$ of order $(n, j)$. We prove that $p_{j, n}^{f}(q)=$ $H_{j, n}^{f}(q)$ by induction on $n$. If $n=0$ we then have to put no numbers in $j+a_{r}$ subsets such that the first $j$ subset are nonempty: this is possible if and only if $j=0$; indeed $H_{0,0}^{f}(q)=1$. If $j=0$ and $n>1$ we can't put $1_{1}, \ldots, 1_{r}$ in one of the first 0 subsets, so there aren't any $f$-Stirling partitions of order $(n, 0)$. Therefore $p_{j, n}^{f}(q)=H_{j, n}^{f}(q)$ if $n=0$ or $j=0$.

Now, suppose $j, n \geq 1$ and the claim true for smaller values. We can obtain an $f$ Stirling partition $P$ either by adding a subset $\left\{n_{1}, \ldots, n_{k}\right\}$ to an $f$-Stirling partition $P_{1}$ of order $(n-1, j-1)$, or by adding each number $n_{i}$ with $1 \leq i \leq r$ to one of the first $j+a_{i}$ subset of an $f$-Stirling partition $P_{2}$ of order $(n-1, j)$.

The generating function for the first case is clearly $p_{j-1, n-1}^{f}(q)$ and the generating function for the latter case is clearly $\left[j+a_{1}\right] \cdots\left[j+a_{r}\right] p_{j, n-1}^{f}(q)$. Now the result follows by adding these two generating functions and using (19). 
For example here are all $f$-Stirling partitions of order $(3,2)$ when $f=(x+1)(x+2)$ :

$\begin{array}{rrrrrr}\text { label } & 1 & 2 & 0 & -1 & \\ P_{1}: & \left\{1_{1}, 1_{2}, 3_{1}, 3_{2}\right\} & \left\{2_{1}, 2_{2}\right\} & \{\} & \{\} & s_{P_{1}}=2 \\ P_{2}: & \left\{1_{1}, 1_{2}, 3_{1}\right\} & \left\{2_{1}, 2_{2}, 3_{2}\right\} & \{\} & \{\} & s_{P_{2}}=1 \\ P_{3}: & \left\{1_{1}, 1_{2}, 3_{1}\right\} & \left\{2_{1}, 2_{2}\right\} & \left\{3_{2}\right\} & \{\} & s_{P_{3}}=3 \\ P_{4}: & \left\{1_{1}, 1_{2}, 3_{1}\right\} & \left\{2_{1}, 2_{2}\right\} & \{\} & \left\{3_{2}\right\} & s_{P_{4}}=4 \\ P_{5}: & \left\{1_{1}, 1_{2}, 3_{2}\right\} & \left\{2_{1}, 2_{2}, 3_{1}\right\} & \{\} & \{\} & s_{P_{5}}=1 \\ P_{6}: & \left\{1_{1}, 1_{2}\right\} & \left\{2_{1}, 2_{2}, 3_{1}, 3_{2}\right\} & \{\} & \{\} & s_{P_{6}}=0 \\ P_{7}: & \left\{1_{1}, 1_{2}\right\} & \left\{2_{1}, 2_{2}, 3_{1}\right\} & \left\{3_{2}\right\} & \{\} & s_{P_{7}}=2 \\ P_{8}: & \left\{1_{1}, 1_{2}\right\} & \left\{2_{1}, 2_{2}, 3_{1}\right\} & \{\} & \left\{3_{2}\right\} & s_{P_{8}}=3 \\ P_{9}: & \left\{1_{1}, 1_{2}, 3_{2}\right\} & \left\{2_{1}, 2_{2}\right\} & \left\{3_{1}\right\} & \{\} & s_{P_{9}}=3 \\ P_{10}: & \left\{1_{1}, 1_{2}\right\} & \left\{2_{1}, 2_{2}, 3_{2}\right\} & \left\{3_{1}\right\} & \{\} & s_{P_{10}}=2 \\ P_{11}: & \left\{1_{1}, 1_{2}\right\} & \left\{2_{1}, 2_{2}\right\} & \left\{3_{1}, 3_{2}\right\} & \{\} & s_{P_{11}}=4 \\ P_{12}: & \left\{1_{1}, 1_{2}\right\} & \left\{2_{1}, 2_{2}\right\} & \left\{3_{1}\right\} & \left\{3_{2}\right\} & s_{P_{12}}=5 \\ \text { label } & 1 & & 0 & -1 & \\ P_{13}: & \left\{1_{1}, 1_{2}, 2_{1}, 2_{2}\right\} & \left\{3_{1}, 3_{2}\right\} & \{\} & \{\} & s_{P_{13}}=0 \\ P_{14}: & \left\{1_{1}, 1_{2}, 2_{1}\right\} & \left\{3_{1}, 3_{2}\right\} & \left\{2_{2}\right\} & \{\} & s_{P_{14}}=1 \\ P_{15}: & \left\{1_{1}, 1_{2}, 2_{1}\right\} & \left\{3_{1}, 3_{2}\right\} & \{\} & \left\{2_{2}\right\} & s_{P_{15}}=2 \\ P_{16}: & \left\{1_{1}, 1_{2}, 2_{2}\right\} & \left\{3_{1}, 3_{2}\right\} & \left\{2_{1}\right\} & \{\} & s_{P_{16}}=1 \\ P_{17}: & \left\{1_{1}, 1_{2}\right\} & \left\{3_{1}, 3_{2}\right\} & \left\{2_{1}, 2_{2}\right\} & \{\} & s_{P_{17}}=2 \\ P_{18}: & \left\{1_{1}, 1_{2}\right\} & \left\{3_{1}, 3_{2}\right\} & \left\{2_{1}\right\} & \left\{2_{2}\right\} & s_{P_{18}}=3\end{array}$

Indeed $H_{2,3}^{(x+1)(x+2)}(q)=2+4 q+5 q^{2}+4 q^{3}+2 q^{4}+q^{5}$. When $r=1$ and $a_{1}=0$, as suggested by the anonymous referee, we recognize the following combinatorial interpretation of $q$-Stirling numbers of the second kind due to Sagan [14, Theorems 4.1 and 1.1].

Corollary 3. For all $j, n, k \in \mathbb{N}, S(n, j)[k]$ is the number of partitions $P$ of $\{1, \ldots, n\}$ in $j$ nonempty blocks with $s_{P}=k$.

When we evaluate all polynomials in $q=1$ we get

Corollary 4. For all $j, n \in \mathbb{N}, H_{j, n}^{f}$ is the number of $f$-Stirling partitions of order $(n, j)$.

In particular, when $r=2, a_{1}=0$ and $a_{2}=1$ (i. e. for the Legendre-Stirling numbers), we obtain the following result.

Corollary 5. For all $n, j, k \in \mathbb{N}$ we have that $L S_{n}^{(j)}[k]$ is the number of $x(x+1)$-Stirling partitions $P$ of order $(n, j)$ such that $s_{P}=k$. 
This interpretation is similar to one in [2, Theorem 2]. Here the authors fill $j+1$ sets with the numbers in (21), such that there exists a set (the "zero box") which is the only set that may be empty and it may not contain both copies of any number; the other $j$ sets are indistinguishable and each is non-empty; each such set contains both copies of its smallest element and does not contain both copies of any other elements. Consider an $x(x+1)$-Stirling partition and for all $m \leq n$ if $A_{m_{1}} \leq A_{m_{2}}$ move the element $m_{1}$ in the set immediately lower than $A_{m_{1}}$. It gives a bijective proof of the equivalence of both interpretations.

Finally, for the Jacobi-Stirling numbers, Corollary 4 becomes:

Corollary 6. For all $j, n, z \in \mathbb{N}, J S_{n}^{(j)}$ is the number of $x(x+z)$-Stirling partitions of $\operatorname{order}(n, j)$.

\subsection{Combinatorial interpretation of $E_{j, n}^{f}[k]$}

In [5], for any cycle of a permutation Egge defines the cycle maxima (resp. cycle minima) as the maximum (resp. minimum) of the numbers in the cycle. We consider an $r$-tuple of permutations $\pi=\left(\pi_{1}, \ldots, \pi_{r}\right)$ with $\pi_{i} \in S_{n+a_{i}}$ for all $i=1, \ldots, r$ and we say that $\pi$ is $f$-Stirling of order $(n, j)$ if and only if

(a) each $\pi_{i}$ has exactly $j+a_{i}$ cycles;

(b) $\pi_{1}, \pi_{2}, \ldots, \pi_{r}$ have the same cycle maxima less than $n+1$;

(c) the orbits of $n, n+1, \ldots, n+a_{i}$ in $\pi_{i}$ are pairwise distinct for all $i \leq r$.

For example, an $f$-Stirling 2-tuple permutation of order $(3,2)$, with $f=(x+1)(x+2)$, is $\{(4)(3,1)(2),(5)(4,1)(3)(2)\}$. The 2-tuples $\sigma_{1}=\{(4)(3,1)(2),(5)(4,1)(2,3)\}, \sigma_{2}=$ $\{(4)(3,1)(2),(5)(4,2)(3)(1)\}$ and $\sigma_{3}=\{(4)(3,1)(2),(5,4)(3)(2)(1)\}$ are not $f$-Stirling since one of the previous conditions fails.

Given a permutation $\rho \in S_{n}$ we define a word in the alphabet $\{1, \ldots, n\}$ as follows: we write each cycle of $\rho$ with the cycle maxima in the first place and we order the cycles by their cycle maxima in decreasing order; then we delete the brackets. We call $s_{\rho}$ this word. For example, if $\rho=(162)(45) \in S_{6}$ then $s_{\rho}=621543$; if $\sigma=(12)(45) \in S_{6}$ then $s_{\sigma}=654321$. Note that we can obtain the same word from two different permutations: for example if $\rho_{1}=(12)(45) \in S_{6}, \rho_{2}=(132)(654) \in S_{6}$ then $s_{\rho_{1}}=s_{\rho_{2}}=654321$. Now let $s$ be a such word in the alphabet $\{1, \ldots, n\}$. Let's define

$$
\operatorname{coinv}_{s}^{(i)}:=\mid\{j \in\{1, \ldots, n\} \mid j>i \text { and } i \text { is on the left of } j \text { in } s\} \mid \text {. }
$$

For example, if $s=623541$ then $\operatorname{coinv}_{s}^{(2)}=3 ; \operatorname{coinv}_{s}^{(5)}=0$. We then set

$$
\operatorname{coinv}_{s}:=\sum_{i=1}^{n} \operatorname{coinv}_{s}^{(i)} .
$$

We call coinversions of $s$ the number $\operatorname{coinv}_{s}$. Equivalently, coinv ${ }_{s}$ is the minimum number of exchanges of two consecutive elements in $s$ to obtain the word $n, n-1, \ldots, 2,1$. 
Proposition 7. Fix $n, j, k \in \mathbb{N}$. Then $E_{j, n}^{f}[k]$ is the number of $f$-Stirling $r$-tuples of permutations $\pi=\left(\pi_{1}, \ldots, \pi_{r}\right)$ such that

$$
\sum_{i=1}^{r} \operatorname{coinv}_{\pi_{i}}=k
$$

Proof. For all $n, j \geq 0$ we consider the following generating function

$$
p_{j, n}^{f}(q):=\sum_{\pi} q^{\sum_{i=1}^{r} \operatorname{coinv}_{\pi_{i}}}
$$

where the sum is over all $f$-Stirling $r$-tuples of permutations $\pi=\left(\pi_{1}, \ldots, \pi_{r}\right)$ satisfying (22). We prove by induction on $n$ that $p_{j, n}^{n}(q)=E_{j, n}^{f}(q)$. If $j=0$, by conditions (a) and (c), we have only one possibility when $n=0$. If $n<j$, condition (a) is never satisfied. Indeed in both cases $E_{j, n}^{f}(q)=0$ except $E_{0,0}^{f}(q)=1$. Therefore, let's suppose $1 \leq j \leq n$.

We can make an $f$-Stirling $r$-tuple of permutations of order $(n, j)$ in two distinct ways: 1 ) start from an $f$-Stirling $r$-tuple of permutations $\pi$ of order $(n-1, j-1)$, increase each number of one unit and then add in each permutation $\pi_{i}$ (with abuse of notations) the trivial cycle (1). In this case, for all $i \leq r \operatorname{coinv}_{\pi_{i}}^{(1)}=0$.

2 ) start from an $f$-Stirling $r$-tuple of permutations $\pi$ of order $(n-1, j)$, increase each number of one unit and then add the element 1 in each permutation $\pi_{i}$. This operation can be done in $n-1+a_{i}$ ways for all permutation $\pi_{i}$ (it is equivalent to put the new number in the word $s_{\pi_{i}}$ in all positions except the first) and $\operatorname{coinv}_{\pi_{i}}^{(1)}$ can assume all values between 0 and $n-2+a_{i}$.

In the case 1) the generating function is clearly $p_{j-1, n-1}^{f}(q)$ and in the case 2) the generating function is given by $\left[n-1+a_{1}\right] \cdots\left[n-1+a_{r}\right] E_{j, n-1}$. Now the result will follow by adding these two generating functions and using (20). 
For example, here are all $f$-Stirling 2 -tuples of permutations of order $(3,2)$ when $f=(x+1)(x+2)$.

$\begin{array}{clll}\sigma_{1}: & (4,1)(3)(2) ; & (5,1)(4)(3)(2) & \text { coinv }=2+3=5 \\ \sigma_{2}: & (4,1)(3)(2) ; & (5)(4,1)(3)(2) & \text { coinv }=2+2=4 \\ \sigma_{3}: & (4,1)(3)(2) ; & (5)(4)(3,1)(2) & \text { coinv }=2+1=3 \\ \sigma_{4}: & (4,1)(3)(2) ; & (5)(4)(3)(2,1) & \text { coinv }=2+0=2 \\ \sigma_{5}: & (4)(3,1)(2) ; & (5,1)(4)(3)(2) & \text { coinv }=1+3=4 \\ \sigma_{6}: & (4)(3,1)(2) ; & (5)(4,1)(3)(2) & \text { coinv }=1+2=3 \\ \sigma_{7}: & (4)(3,1)(2) ; & (5)(4)(3,1)(2) & \text { coinv }=1+1=2 \\ \sigma_{8}: & (4)(3,1)(2) ; & (5)(4)(3)(2,1) & \text { coinv }=1+0=1 \\ \sigma_{9}: & (4)(3)(2,1) ; & (5,1)(4)(3)(2) & \text { coinv }=0+3=3 \\ \sigma_{10}: & (4)(3)(2,1) ; & (5)(4,1)(3)(2) & \text { coinv }=0+2=2 \\ \sigma_{11}: & (4)(3)(2,1) ; & (5)(4)(3,1)(2) & \text { coinv }=0+1=1 \\ \sigma_{12}: & (4)(3)(2,1) ; & (5)(4)(3)(2,1) & \text { coinv }=0+0=0 \\ \sigma_{13}: & (4,2)(3)(1) ; & (5,2)(4)(3)(1) & \text { coinv }=1+2=3 \\ \sigma_{14}: & (4,2)(3)(1) ; & (5)(4,2)(3)(1) & \text { coinv }=1+1=2 \\ \sigma_{15}: & (4,2)(3)(1) ; & (5)(4)(3,2)(1) & \text { coinv }=1+0=1 \\ \sigma_{16}: & (4)(3,2)(1) ; & (5,2)(4)(3)(1) & \text { coinv }=0+2=2 \\ \sigma_{17}: & (4)(3,2)(1) ; & (5)(4,2)(3)(1) & \text { coinv }=0+1=1 \\ \sigma_{18}: & (4)(3,2)(1) ; & (5)(4)(3,2)(1) & \text { coinv }=0+0=0\end{array}$

Indeed, $E_{2,3}^{(x+1)(x+2)}(q)=2+4 q+5 q^{2}+4 q^{3}+2 q^{4}+q^{5}$. When $r=1$ and $a_{1}=0$, with the same arguments of the proof of Proposition 7, we have the following new combinatorial interpretation of $q$-Stirling numbers of the first kind.

Corollary 8. For all $j, n, k \in \mathbb{N}, S(n, j)[k]$ is the number of permutations $\rho \in S_{n}$ with $j$ cycles with coinv $_{s_{\rho}}=k$.

When we evaluate all polynomials in $q=1$ we have

Corollary 9. For all $j, n \in \mathbb{N}, E_{j, n}^{f}$ is the number of $f$-Stirling $r$-tuples of permutations of order $(n, j)$.

For $r=2, a_{1}=0$ and $a_{2}=1$, Corollary 9 reduces to [5, Theorem 2.5].

\section{Combinatorial interpretations as polynomials}

In this section we consider the numbers $H_{j, n}^{f}$ and $E_{j, n}^{f}$ as polynomials in $a_{1}, \ldots, a_{r}$ with $r$ a fixed positive integer and $f(x)=\left(x+a_{1}\right) \cdots\left(x+a_{r}\right)$. 


\subsection{Combinatorial interpretation of $H_{j, n}^{f}$}

Fix $j, n \in \mathbb{N}$ and consider exactly $r$ copies of numbers $0, \ldots, n$, i. e.

$$
0_{1}, 0_{2}, \ldots, 0_{r}, 1_{1}, 1_{2}, \ldots, 1_{r}, \ldots \ldots, n_{1}, n_{2}, \ldots, n_{r} .
$$

We consider a partition $P$ of $(23)$ and we say that $P$ is $r$-Stirling of order $(n, j)$ if $P$ is a partition of (23) into $j+1$ subsets such that

- all the $j+1$ subsets are nonempty;

- each subset contains the minimum number with all its indices;

- if $n \neq 0$ then no subset contains both $0_{i}, 1_{i}$ for any index $i \leq r$.

Given such a partition $P$, we call 0 -subset of $P$ the only subset that contains $0_{1}, \ldots, 0_{r}$.

For example, a 2-Stirling partition of order $(3,2)$ is $\left(\left\{0_{1}, 0_{2}, 3_{2}\right\},\left\{1_{1}, 1_{2}, 3_{1}\right\}\right.$, $\left.\left\{2_{1}, 2_{2}\right\}\right)$. The partitions $P_{1}=\left(\left\{0_{1}, 0_{2}, 2_{1}, 2_{2}\right\},\left\{1_{1}, 1_{2}, 3_{1}, 3_{2}\right\},\{\}\right), P_{2}=\left(\left\{0_{1}, 0_{2}, 1_{1}\right\}\right.$, $\left.\left\{1_{2}, 2_{1}, 2_{2}\right\},\left\{3_{1}, 3_{2}\right\}\right)$ and $P_{3}=\left(\left\{0_{1}, 0_{2}, 1_{1}, 1_{2}\right\},\left\{2_{1}, 2_{2}\right\},\left\{3_{1}, 3_{2}\right\}\right)$ are not 2-Stirling since one of the previous condition fails.

Proposition 10. For all $j, n \in \mathbb{N}$ and for all $\beta_{1}, \ldots, \beta_{r} \in \mathbb{N}$ we have that the coefficient of $a_{1}^{\beta_{1}} a_{2}^{\beta_{2}} \cdots a_{r}^{\beta_{r}}$ in $H_{j, n}^{f}$ is the number of $r$-Stirling partitions of order $(n, j)$ whose 0 -subset contains $\beta_{i}+1$ numbers with index $i$ for all $i \in\{1, \ldots, r\}$.

Proof. For all $n, j \geq 0$ we consider the following generating function

$$
p_{j, n}\left(a_{1}, \ldots, a_{r}\right):=\sum_{P} a_{1}^{P(1)} \cdots a_{r}^{P(r)}
$$

where the sum is over all $r$-Stirling partitions of order $(n, j)$ and $P(i)+1$ is the number of elements in the 0 -subset having label $i$, for $i=1, \ldots, r$. We will prove by induction that $p_{j, n}\left(a_{1}, \ldots, a_{r}\right)=H_{j, n}^{f}$. We set $p_{j, n}\left(\beta_{1}, \ldots, \beta_{r}\right)$ the number of $r$-Stirling partitions $P$ of order $(n, j)$ such that the 0 -subset of $P$ contains $\beta_{i}+1$ numbers with index $i$ for all $i \in\{1, \ldots, r\}$. If $n=0$ then we put the numbers $0_{1}, \ldots, 0_{r}$ in the same subset. Therefore there exist $r$-Stirling partitions of order $(0, j)$ if and only if $j=0$. Indeed, we have $H_{0, j}^{f}=\delta_{0, j}$. If $j=0$, since we cannot put $0_{1}, \ldots, 0_{r}, 1_{1}, \ldots, 1_{r}$ in the same subset, then necessarily $n=0$.

Now, suppose that $j, n \geq 1$ and the claim is true for smaller values. We can obtain an $r$-Stirling partition $P$ or by adding a subset $\left\{n_{1}, \ldots, n_{r}\right\}$ to an $r$-Stirling partition $P_{1}$ of order $(n-1, j-1)$, or by adding each number $n_{i}$ with $1 \leq i \leq r$ in one of the $j+1$ subsets of an $r$-Stirling partition $P_{2}$ of order $(n-1, j)$. The generating function for the first case is clearly $p_{j-1, n-1}\left(a_{1}, \ldots, a_{r}\right)$ and the generating function for the second case is clearly $\left(j+a_{1}\right) \cdots\left(j+a_{r}\right) p_{j, n-1}\left(a_{1}, \ldots, a_{r}\right)$. Now the result follows by adding these two generating functions and using (4) and (14). 
For example, here are all 2-Stirling partitions of order $(3,2)$.

$\begin{array}{rrrr}P_{1}: & \left\{0_{1}, 0_{2}, 3_{1}, 3_{2}\right\} & \left\{1_{1}, 1_{2}\right\} & \left\{2_{1}, 2_{2}\right\} \\ P_{2}: & \left\{0_{1}, 0_{2}, 3_{1}\right\} & \left\{1_{1}, 1_{2}, 3_{2}\right\} & \left\{2_{1}, 2_{2}\right\} \\ P_{3}: & \left\{0_{1}, 0_{2}, 3_{1}\right\} & \left\{1_{1}, 1_{2}\right\} & \left\{2_{1}, 2_{2}, 3_{2}\right\} \\ P_{4}: & \left\{0_{1}, 0_{2}, 3_{2}\right\} & \left\{1_{1}, 1_{2}, 3_{1}\right\} & \left\{2_{1}, 2_{2}\right\} \\ P_{5}: & \left\{0_{1}, 0_{2}\right\} & \left\{1_{1}, 1_{2}, 3_{1}, 3_{2}\right\} & \left\{2_{1}, 2_{2}\right\} \\ P_{6}: & \left\{0_{1}, 0_{2}\right\} & \left\{1_{1}, 1_{2}, 3_{1}\right\} & \left\{2_{1}, 2_{2}, 3_{2}\right\} \\ P_{7}: & \left\{0_{1}, 0_{2}, 3_{2}\right\} & \left\{1_{1}, 1_{2}\right\} & \left\{2_{1}, 2_{2}, 3_{1}\right\} \\ P_{8}: & \left\{0_{1}, 0_{2}\right\} & \left\{1_{1}, 1_{2}, 3_{2}\right\} & \left\{2_{1}, 2_{2}, 3_{1}\right\} \\ P_{9}: & \left\{0_{1}, 0_{2}\right\} & \left\{1_{1}, 1_{2}\right\} & \left\{2_{1}, 2_{2}, 3_{1}, 3_{2}\right\} \\ P_{10}: & \left\{0_{1}, 0_{2}, 2_{1}, 2_{2}\right\} & \left\{1_{1}, 1_{2}\right\} & \left\{3_{1}, 3_{2}\right\} \\ P_{11}: & \left\{0_{1}, 0_{2}, 2_{1}\right\} & \left\{1_{1}, 1_{2}, 2_{2}\right\} & \left\{3_{1}, 3_{2}\right\} \\ P_{12}: & \left\{0_{1}, 0_{2}, 2_{2}\right\} & \left\{1_{1}, 1_{2}, 2_{1}\right\} & \left\{3_{1}, 3_{2}\right\} \\ P_{13}: & \left\{0_{1}, 0_{2}\right\} & \left\{1_{1}, 1_{2}, 2_{1}, 2_{2}\right\} & \left\{3_{1}, 3_{2}\right\}\end{array}$

Indeed, $H_{2,3}^{f}=5+3\left(a_{1}+a_{2}\right)+2 a_{1} a_{2}$.

In the case of Jacobi-Stirling numbers we have $f=x(x+z)$ : then, in our notation, we have $r=2, a_{1}=0$, and $a_{2}=z$. To set $a_{1}=0$ is equivalent to take only the monomials without factor $a_{1}$. By Proposition 10, we have to consider only the 2-Stirling partitions $P$ of order $(n, j)$ of $0_{1}, 0_{2}, \ldots, n_{1}, n_{2}$ with the 0 -subset without numbers labeled by 1 except $0_{1}$. With this remark our interpretation in Proposition 10 reduces to

Corollary 11. For all $j, n, \beta \in N$ the coefficient of $z^{\beta}$ in $J S_{j, n}(z)$ is the number of 2Stirling partitions of order $(n, j)$ whose 0 -subset contains $\beta+1$ numbers with index 2 and only one number with index 1 (necessarily $0_{1}$ ).

This result is equivalent of the one given in [8, Theorem 2]. Here the authors consider partitions of (23) such that each set contains both copies of its smallest element and does not contain both copies of any other number. Consider a 2-Stirling partition whose 0 -subset has no positive numbers labeled by 1 . If $m_{1}, m_{2}$ are in the same set, with $1 \leq m \leq n$, then move $m_{1}$ in the 0 -subset. This prove that the two interpretations are equivalent.

\subsection{Combinatorial interpretation of $E_{j, n}^{f}$}

Fix $j, n \in \mathbb{N}$ and consider an $r$-tuple of permutations $\pi=\left(\pi_{1}, \ldots, \pi_{r}\right) \in S_{n+1}^{r}$. We say that $\pi$ is $r$-Stirling of order $(n, j)$ if and only if

(a') each $\pi_{i}$ has exactly $j+1$ cycles;

(b') $\pi_{1}, \pi_{2}, \ldots, \pi_{r}$ have the same cycle minima; 
(c') if $n \neq 0$ the orbits of 1 and 2 are disjoint for all permutations $\pi_{i}$.

For example, a 2-Stirling 2-tuple of permutations of order $(3,2)$ is $\{(1)(2,4)(3)$, $(1)(2)(3,4)\}$. The 2-tuples $\sigma_{1}=\{(1)(2,4)(3),(1)(2,3)(4)\}$ and $\sigma_{2}=\{(1,2)(3)(4)$, $(1,2)(3)(4)\}$ are not 2-Stirling since one of the previous condition fails.

Given a word $w=w(1) \ldots w(l)$ on the finite alphabet $\{1, \ldots, n+1\}$, a letter $w(j)$ is a left-to-right minimum of $w$ if $w(k)>w(j)$ for every $k \in\{1, \ldots, j-1\}$. We define $\operatorname{lrm}(w)$ to be the number of left-to-right minima of $w$ and given $\pi \in S_{n+1}$ we define $\operatorname{lrm}(\sigma)=\operatorname{lrm}\left(\sigma(1), \sigma^{2}(1), \ldots, 1\right)$ (the elements are only in the orbit of 1 ).

Proposition 12. For all $n, j \in \mathbb{N}$ and for all $\beta_{1}, \ldots, \beta_{r} \in \mathbb{N}$ the coefficient of $a_{1}^{\beta_{1}} a_{2}^{\beta_{2}} \cdots a_{r}^{\beta_{r}}$ in $E_{j, n}^{r}$ is the number of $r$-Stirling $r$-tuples of permutations $\pi=\left(\pi_{1}, \ldots, \pi_{r}\right)$ such that $\operatorname{lrm}\left(\pi_{i}\right)=\beta_{i}+1$ for all $i=1, \ldots, r$.

Proof. For all $n, j \geq 0$ we consider the following generating function

$$
p_{j, n}\left(a_{1}, \ldots, a_{r}\right):=\sum_{\pi} a_{1}^{\operatorname{lrm}\left(\pi_{1}\right)-1} \cdots a_{r}^{\operatorname{lrm}\left(\pi_{r}\right)-1}
$$

where the sum is over all $r$-Stirling $r$-tuples of permutations $\pi=\left(\pi_{1}, \ldots, \pi_{r}\right)$ of order $(n, j)$. We will prove by induction that $p_{j, n}\left(a_{1}, \ldots, a_{r}\right)=E_{j, n}^{f}$. If $j=0$, by conditions (a') and $\left(c^{\prime}\right)$, we have only one $r$-tuple of $r$-Stirling permutations of order $(n, 0)$ when $n=0$ and no one when $n>0$. If $n<j$, condition ( $\left.\mathrm{a}^{\prime}\right)$ is never satisfied. Indeed, in both cases $E_{j, n}^{f}=0$ except $E_{0,0}=1$. Let's now suppose that $1 \leq j \leq n$. We can construct a $r$-Stirling $r$-tuples of permutations $\pi=\left(\pi_{1}, \ldots, \pi_{r}\right)$ of order $(n, j)$ in two different ways (and the reader can check that in these ways we obtain all such $r$-tuples). The first possibility is to start with an $r$-Stirling $r$-tuple of permutations of order $(n-1, j-1)$ and add a trivial cycle $(n+1)$ in each its permutation. In this case we preserve the orbit of $(1)$, i. e. the numbers of left-to-right minima of all permutations does not change.

The second possibility is to start with an $r$-Stirling $r$-tuple $\pi^{\prime}=\left(\pi_{1}^{\prime}, \ldots, \pi_{r}^{\prime}\right)$ of permutations of order $(n-1, j)$ and add the number $n+1$ in each permutation $\pi_{i}^{\prime}$. In this case we increase the numbers of left-to-right minima if and only if we put $n+1$ on the right of 1 in the cycle notation of $\pi_{i}$, i. e. $\pi_{i}^{\prime}(1)=n+1, \pi_{i}^{\prime}(n+1)=\pi_{i}(1)$ and $\pi^{\prime}(i)=\pi(i)$ otherwise. The generating function for the first case is clearly $p_{j-1, n-1}\left(a_{1}, \ldots, a_{r}\right)$ and the generating function for the second case in clearly $\left(a_{1}+n-1\right) \cdots\left(a_{r}+n-1\right) p_{j, n-1}$. Now the result follows by adding these two generating functions and using (5) and (15). 
For example, here are all 2-Stirling 2-tuples of permutations of order $(3,2)$.

$\begin{array}{rlll}\sigma_{1}: & (1,4)(2)(3) ; & (1,4)(2)(3) & \operatorname{lrm}=(2,2) \\ \sigma_{2}: & (1,4)(2)(3) ; & (1)(2,4)(3) & \operatorname{lrm}=(2,1) \\ \sigma_{3}: & (1,4)(2)(3) ; & (1)(2)(3,4) & \operatorname{lrm}=(2,1) \\ \sigma_{4}: & (1)(2,4)(3) ; & (1,4)(2)(3) & \operatorname{lrm}=(1,2) \\ \sigma_{5}: & (1)(2,4)(3) ; & (1)(2,4)(3) & \operatorname{lrm}=(1,1) \\ \sigma_{6}: & (1)(2,4)(3) ; & (1)(2)(3,4) & \operatorname{lrm}=(1,1) \\ \sigma_{7}: & (1)(2)(3,4) ; & (1,4)(2)(3) & \operatorname{lrm}=(1,2) \\ \sigma_{8}: & (1)(2)(3,4) ; & (1)(2,4)(3) & \operatorname{lrm}=(1,1) \\ \sigma_{9}: & (1)(2)(3,4) ; & (1)(2)(3,4) & \operatorname{lrm}=(1,1) \\ \sigma_{10}: & (1,3)(2)(4) ; & (1,3)(2)(4) & \operatorname{lrm}=(2,2) \\ \sigma_{11}: & (1,3)(2)(4) ; & (1)(2,3)(4) & \operatorname{lrm}=(2,1) \\ \sigma_{12}: & (1)(2,3)(4) ; & (1,3)(2)(4) & \operatorname{lrm}=(1,2) \\ \sigma_{13}: & (1)(2,3)(4) ; & (1)(2,3)(4) & \operatorname{lrm}=(1,1)\end{array}$

We have 5 couples of permutations with left-to-right minima $(1,1), 3$ with left-to-right minima $(2,1)$ and $(1,2)$ and 2 with left-to-right minima $(2,2)$. Indeed, $E_{2,3}^{f}=5+3\left(a_{1}+\right.$ $\left.a_{2}\right)+2 a_{1} a_{2}$.

In the case of Jacobi-Stirling numbers we have $f=x(x+z)$. Therefore, in our notation, we have $r=2, a_{1}=0$, and $a_{2}=z$. To set $a_{1}=0$ is equivalent to take only the monomials without factor $a_{1}$. By Proposition 12, we have to consider only the 2-Stirling 2-tuples of permutations $\pi=\left(\pi_{1}, \pi_{2}\right)$ with the orbit of 1 in $\pi_{1}$ be trivial. Therefore, Proposition 12 reduces to

Corollary 13. For all $n, j, \beta \in \mathbb{N}$ the coefficient of $z^{\beta}$ in $J c_{j, n}(z)$ is the number of 2Stirling 2-permutations $\left(\pi_{1}, \pi_{2}\right)$ such that $\pi_{1}(1)=1$ and $\operatorname{lrm}\left(\pi_{2}\right)=\beta+1$.

Obviously, $\pi_{1}$ can be identified with a permutation of $S_{n}$. With this identification, our interpretation is the same of the one given in [8, Theroem 7].

\section{Developments on some generalization of symmetric functions}

In this section we analyze other functions introduced in $[3,9]$ which generalize the elementary and complete symmetric functions. We give a combinatorial interpretation if we evaluate them in $f(1), f(2), \ldots$ as done in the previous sections. In particular, this approach is used to obtain a new interpretation of the Jacobi-Stirling numbers.

In [9, Section 7] Gessel and Viennot introduce the generalized Schur functions, defined on a set $X=\left\{x_{1}, \ldots, x_{n}\right\}$ of indeterminates, with an order relation $\mathrm{R}$, in the following 
way

$$
h_{k}^{\mathrm{R}}\left(x_{1}, \ldots, x_{n}\right):=\sum_{i_{1}, \ldots, i_{k}} x_{i_{1}} x_{i_{2}} \cdots x_{i_{k}}
$$

where the sum is over all indices such that $i_{1} \mathrm{R} i_{2} \mathrm{R} \cdots \mathrm{R} i_{k}$. If $k=0$ then $h_{0}^{\mathrm{R}}=0$. If we consider the classical order relations $<$ or $\leq$ we have the elementary or complete symmetric functions. In [3, Section 5] the author turns his attention to the relation $\mathrm{R}_{t}$ defined as follows: for all $t \in \mathbb{N}$ and for all $i, j \in \mathbb{N}$, we set $x_{i} \mathrm{R}_{t} x_{j}$ if and only if $j-i \geq t$. Another generalization of the elementary symmetric functions is given in [3, Section 5] as follows: for all $t, n, k \in \mathbb{P}$ we set

$$
a_{k}^{(t)}\left(x_{1}, \ldots, x_{n}\right):=\sum_{i_{1}, \ldots, i_{k}} x_{i_{1}} x_{i_{2}} \cdots x_{i_{k}}
$$

where the sum is over all $1 \leq i_{1}<\cdots<i_{k} \leq n$ such that $i_{j} \equiv j(\bmod t)$ for all $j=1, \ldots, k$. For example, $a_{2}^{(2)}\left(x_{1}, \ldots, x_{6}\right)=x_{1} x_{2}+x_{1} x_{4}+x_{1} x_{6}+x_{3} x_{4}+x_{3} x_{6}+x_{5} x_{6}$.

In the next two subsections we give combinatorial interpretations of

$h_{k}^{\mathrm{R}_{t}}(f(1), \ldots, f(n))$ and $a_{k}^{(t)}(f(1), \ldots, f(n))$, where $f$ is the polynomial with all real zeros and nonnegative coefficients.

\subsection{Combinatorial interpretation of $h_{n}^{\mathrm{R}_{t}}$}

Given $r \in \mathbb{N}, a_{1}, \ldots, a_{r} \in \mathbb{N}$, let $f$ be the polynomial $f(x)=\left(x+a_{1}\right) \cdots\left(x+a_{r}\right)$. Let $n \in \mathbb{N}$ and $\sigma \in S_{n}$ be a permutation. We decompose $\sigma$ in disjoint cycles and we write each cycle with its minimum at the first place. Then we label each cycle with its minimum and order the cycles in increasing order of their minima. Therefore, examples of decompositions are (136)(25)(4) and (1652)(34). We say that two or more permutations have the same ordered cycle structure if in the previous notation, the sequences of lengths of the cycles are equals (in the following we denote by $l(c)$ the length of a cycle $c$ ). For example, (136)(25)(4) and $(123)(45)(6)$ have the same ordered cycle structure, while $(136)(25)(4)$ and $(15)(234)(6)$ no.

We say that one or more permutations have the same ordered cycle structure up to $k$ if the first $k$ elements of the sequences of the lengths of the cycles are equals for all permutations and the lengths of remaining cycles (if they exist) are equal to 1 . For example $(132)(45)$ and $(176)(24)(3)(5)$ have the same ordered cycle structure up to 2 , $(132)(45)$ and $(176)(24)(35)$ no.

Now fix a permutation $\sigma$ with ordered cycles $c_{i_{1}}, \ldots, c_{i_{r}}$ for some integer $r \in \mathbb{N}$. Each cycle is labeled by its minimum element. We define a distance between two cycles via the following natural definition

$$
d\left(c_{i}, c_{j}\right):=\mid\left\{c_{k} \text { cycle of } \sigma \mid i<k \leq j\right\} \mid,
$$

if $i \leq j$, else $d\left(c_{i}, c_{j}\right):=d\left(c_{j}, c_{i}\right)$. It is obvious that if two permutations have the same ordered cycle structure, then the distances between corresponding disjoint cycles are the same. Now we can show the following result. 
Proposition 14. Let $r \in \mathbb{P}, a_{1}, \ldots, a_{r} \in \mathbb{N}$ and $f(x)=\left(x+a_{1}\right) \cdots\left(x+a_{r}\right)$. Then for all $n, k \in \mathbb{N}$ and $t \geq 2, h_{n}^{\mathrm{R}_{t}}(f(1), \ldots, f(k))$ is the number of elements in $S_{k+1+a_{1}} \times \cdots \times S_{k+1+a_{r}}$ such that all permutations have the same ordered cycle structure up to $k+1-n$, each one with $k+1-n+a_{i}$ cycles and length at most 2 and if $c_{i}, c_{j}$ are two cycles with $l\left(c_{i}\right)=l\left(c_{j}\right)=2$ then $d\left(c_{i}, c_{j}\right) \geq t-1$.

Proof. If $n=0$ then each permutation in $S_{k+1+a_{i}}$ as in the statement has $k+1+a_{i}$ disjoint cycles. Therefore it is the trivial permutation, indeed $h_{0}^{\mathrm{R}_{t}}(f(1), \ldots, f(k))=1$. Moreover, if $k<n$ then $k+1-n \leq 0$ and therefore, since the permutations have the same ordered cycle structure up to $k+1-n$, all cycles are trivial; but in this case $k+1-n+a_{i}=k+1+a_{i}$ and this is impossible. Indeed, in this case $h_{n}^{\mathrm{R} t}(f(1), \ldots, f(t))=0$.

Let's suppose that $n \geq 1, k \geq n$ and the thesis true for smaller values of $n$. We fix an $r$-tuple of permutations as in the statement and we turn our attention to the element 1 in each permutation. By assumption, all the first cycles have the same length, in particular they are all trivial or they have length 2 . In the first case we delete the trivial cycle (1) in each permutation and decrease all other elements by one. Therefore we have elements in $S_{k+a_{1}} \times \cdots \times S_{k+a_{r}}$ whose permutations have the same ordered cycle structure up to $k-n$ and $k-n+a_{i}$ cycles. By induction, their number is $h_{n}^{\mathrm{R}_{t}}(f(1), \ldots, f(k-1))$. In the second case, the first cycle of the $i$-th permutation contains a number in $\left\{2, \ldots, k+1+a_{i}\right\}$. In all permutations we delete the first cycle (there are $\prod_{i=1}^{r}\left(k+a_{i}\right)=f(k)$ different ways to have such deleted cycles) and rename all remaining numbers preserving the natural order of them. We get permutations in $S_{k-1+a_{i}}$ with $k-n+a_{i}$ cycles. Moreover, by assumption in each permutation the first $t-2$ cycles are trivial (check the distances) and then we can delete them. We get therefore permutations in $S_{(k-t)+1+a_{i}}$ with $(k-t)-(n-1)+1+a_{i}$ cycles. Their number is by induction $h_{n-1}^{\mathrm{R}_{t}}(f(1), \ldots, f(k-t))$.

By definition, it is simple to check that for all $n, k \geq 1$

$$
h_{n}^{\mathrm{R}_{t}}\left(x_{1}, \ldots, x_{k}\right)=h_{n}^{\mathrm{R}_{t}}\left(x_{1}, \ldots, x_{k-1}\right)+x_{k} h_{n-1}^{\mathrm{R}_{t}}\left(x_{1}, \ldots, x_{k-t}\right) .
$$

This complete the proof.

For example, let $f(x)=x(x+1), t=2, k=3, n=2$. It is simple to check that $h_{2}^{\mathrm{R}_{2}}(f(1), f(2), f(3))=f(1) f(3)=24$. We want obtain elements in $S_{4} \times S_{5}$ as in the previous statement. The first permutation will be (12)(34), (13)(24) or (14)(23); the second permutation, which has the same ordered cycle structure up to 2 of the first, will be one of (12)(34)(5), (12)(35)(4), (13)(24)(5), (13)(25)(4), (14)(23)(5), (14)(25)(3), $(15)(23)(4),(15)(24)(3)$. Indeed, we have 24 possibilities.

If $f(x)=x(x+1), t=3, k=4, n=2$, then $h_{2}^{\mathrm{R}_{3}}(f(1), f(2), f(3), f(4))=f(1) f(4)=$ 40. In this case we want elements in $S_{5} \times S_{6}$ as in the statement. The first permutation is one between $(12)(3)(45),(13)(2)(45),(14)(2)(35)$ and $(15)(2)(34)$; the second permutation is one between (12)(3)(45)(6), (12)(3)(46)(5), (13)(2)(45)(6), (13)(2)(46)(5), $(14)(2)(35)(6),(14)(2)(36)(5),(15)(2)(34)(6)$, (15)(2)(36)(4), (16)(2)(34)(5), (16)(2)(35)(4). We have 40 possibilities.

In [3] we find the polynomials $\bar{h}_{n}^{\mathrm{R}_{t}}\left(x_{1}, \ldots, x_{k}\right)=\sum_{i_{1}, \ldots, i_{r}} x_{i_{1}} \cdots x_{i_{r}}$, that are the same as $h_{n}^{\mathrm{R}_{t}}$ but with another condition $i_{1} \geq t$. In this case, the interpretation is the same as 
that in Proposition 14 but the ordered cycle structure is up to $k-n-t+2$. The proof is essentially the same.

It is possible to consider $h_{n}^{\mathrm{R}_{t}}(f(1), \ldots, f(k))$ as polynomial in $a_{1}, \ldots, a_{r}$. For this purpose we introduce the following definition. Given a permutation $\sigma$ we say that a number $n$ is a big number if in the expansion of $\sigma$ in ordered disjoint cycles, $n$ is greater than each number on its right. For example in $(15)(24)(3)$ the big numbers are $5,4,3$; in $(14)(25)(3)$ the big numbers are 5,3 .

Proposition 15. Let $r \in \mathbb{P}, f(x)=\left(x+a_{1}\right) \cdots\left(x+a_{r}\right)$ and $\beta_{1}, \ldots, \beta_{r} \in \mathbb{N}$. Then for all $n, k \in \mathbb{N}$ and $t \geq 2$, the coefficient of $a_{1}^{\beta_{1}} \cdots a_{r}^{\beta_{r}}$ in $h_{n}^{\mathrm{R}_{t}}(f(1), \ldots, f(k))$ is the number of elements in $S_{k+2}^{r}$ such that all permutations have the same ordered cycle structure up to $k+1-n$, each one with $k+2-n$ cycles and length at most 2 ; if $c_{i}, c_{j}$ are two cycles with $l\left(c_{i}\right)=l\left(c_{j}\right)=2$ then $d\left(c_{i}, c_{j}\right) \geq t-1$ and for all $i=1, \ldots, r$, the $i$-th permutation has $\beta_{i}+1$ big numbers.

We omit the proof which is similar to that of Proposition 14. The only difference is in the case with all the first cycles having lengths 2 . When the maximum number is in the first cycle of the $i$-th permutation (so this number is big), we have a contribution of the indeterminate $a_{i}$.

\subsection{Combinatorial interpretation of $a_{n}^{(t)}$}

In this subsection we give a combinatorial interpretation of $a_{n}^{(t)}(f(1), \ldots, f(k))$, where $f$ is as usual. For permutations, we use the same notation introduced in the previous subsection.

Proposition 16. Let $r \in \mathbb{P}, a_{1}, \ldots, a_{n} \in \mathbb{N}$ and $f(x)=\left(x+a_{1}\right) \cdots\left(x+a_{r}\right)$. Then for all $n, k \in \mathbb{N}$ and $t \geq 1 a_{n}^{(t)}(f(1), \ldots, f(k))$ is the number of elements in $S_{k+1+a_{1}} \times \cdots \times S_{k+1+a_{r}}$ such that all permutations have the same ordered cycle structure up to $k+1-n$, each one with $k+1-n+a_{i}$ cycles and if the $j$-th cycle is not trivial then $k+1-n \equiv j \bmod t$.

Proof. If $n=0$ then each permutation in $S_{k+1+a_{i}}$ as in the statement has $k+1+a_{i}$ disjoint cycles, then it is the trivial permutation, indeed $a_{0}^{t}(f(1), \ldots, f(k))=1$. Moreover, if $k<n$ then $k+1-n \leq 0$ and therefore, by hypothesis of ordered cycle structure up to $k+1-n$, all cycles are trivial; but in this case $k+1-n+a_{i}=k+1+a_{i}$ and this is impossible. Indeed, in this case $a_{n}^{(t)}(f(1), \ldots, f(k))=0$.

Now let $n \geq 1, k \geq n$ and the thesis true for smaller values of $n$. We fix an $r$ tuple of permutation as in the statement and we analyze the cycles labeled by 1 . If $k \neq n \bmod t$ then they are all trivial, and we delete them. In this way we may obtain $a_{n}^{(t)}(f(1), \ldots, f(k-1))$ different $r$-tuples by induction. If $k \equiv n \bmod t$, then the cycles labeled by 1 have arbitrary lengths (but all the same). Let we assume that these lengths are equal to $h+1$ with $h \geq 0$. Then for all $i \leq r$ the first cycle in the $i$-th permutation can be choosen between $\left(k+a_{i}\right)\left(k+a_{i}-1\right) \cdots\left(k+a_{i}-h+1\right)$ different cycles (only one choose if $h=0)$. By multiplying over $i$ we get $f(k) \cdots f(k-h+1)$. We delete now all 
cycles labeled by 1 and so we have elements in $S_{k-h+a_{1}} \times \cdots \times S_{k-h+a_{r}}$ that by induction are $a_{n-h}^{(t)}(f(1), \ldots, f(k-h-1))$.

It is simple to check by definition that $a_{n}^{(t)}\left(x_{1}, \ldots, x_{k}\right)=a_{n}^{(t)}\left(x_{1}, \ldots, x_{k-1}\right)$ if $k \not \equiv n$ $\bmod t$ and that if $n \equiv k \bmod t$ then

$a_{n}^{(t)}\left(x_{1}, \ldots, x_{k}\right)=\sum_{h \geq 0} x_{k} x_{k-1} \cdots x_{k-h+1} a_{n-h}^{(t)}\left(x_{1}, \ldots, x_{k-h-1}\right)$. Thus the proof is completed.

If $t=1, a_{n}^{(1)}\left(x_{1}, \ldots, x_{k}\right)=e_{n}\left(x_{1}, \ldots, x_{k}\right)$. We then have

Corollary 17. For all $n, k \in \mathbb{N}, e_{n}(f(1), \ldots, f(k))$ is the number of elements in $S_{k+1+a_{1}} \times$ $\cdots \times S_{k+1+a_{r}}$ such that all permutations have the same ordered cycle structure up to $k+1-n$, each one with $k+1-n+a_{i}$ cycles.

In the particular case of the Jacobi-Stirling numbers of the first kind, by (18) we have the following new interpretation.

Corollary 18. For $n, k, z \in \mathbb{N} J c(n, k)$ is the number of elements in $S_{n} \times S_{n+z}$ whose permutations have the same ordered cycle structure up to $k$ and the number of cycles are respectively $k$ and $k+z$.

Obviously, we can apply Corollary 18 to the Legendre-Stirling numbers of the first kind just by setting $z=1$. For example, we know that $L c(3,2)=8$. Indeed, by Corollary 18 we obtain the following elements of $S_{3} \times S_{4}$ : (12)(3), (12)(3)(4); (12)(3), (13)(2)(4); $(12)(3),(14)(2)(3) ;(13)(2),(12)(3)(4) ;$

$(13)(2),(13)(2)(4) ;(13)(2),(14)(2)(3) ;(1)(23),(1)(23)(4) ; \quad(1)(23),(1)(24)(3)$.

It is possible to consider $a_{n}^{(t)}(f(1), \ldots, f(k))$ as polynomial in $a_{1}, \ldots, a_{r}$.

Proposition 19. Let $r \in \mathbb{P}, \beta_{1}, \ldots, \beta_{n} \in \mathbb{N}$ and $f(x)=\left(x+a_{1}\right) \cdots\left(x+a_{r}\right)$. Then for all $n, k \in \mathbb{N}$ and $t \geq 1$ the coefficient of $a_{1}^{\beta_{1}} \cdots a_{r}^{\beta_{r}}$ in $a_{n}^{(t)}(f(1), \ldots, f(k))$ is the number of elements in $S_{k+2}^{r}$ such that all permutations have the same ordered cycle structure up to $k+1-n$, each one with $k+2-n$ cycles and if the $j$-th cycle is not trivial then $k+1-n \equiv j$ mod $t$; moreover, the $i$-th permutation has exactly $\beta_{i}+1$ big numbers.

The proof is the same as the one in Proposition 16. In the $i$-th permutation each big number give us a contribution of the indeterminate $a_{i}$.

In the case of the Jacobi-Stirling numbers, we consider the polynomial $f(x)=(x+$ $\left.a_{1}\right)(x+z)$ and consider only the monomial without $a_{1}$ (it is equivalent to set $a_{1}=0$ ). Then we have exactly one big number in the first permutation of $S_{k+2}^{2}$ as in Proposition 19. This number is $k+2$ and it is necessarily in a trivial cycle. Therefore we can omit it and by (18) we have the following result.

Corollary 20. For all $n, j, b \in \mathbb{N}$ the coefficient of $z^{b}$ in $J c(n, j)$ is the number of elements in $S_{n} \times S_{n+1}$ such that both permutations have the same ordered cycle structure up to $j$, with respectively $j$ and $j+1$ cycles and the second permutation has exactly $k+1$ big numbers. 
For example, if $n=3, j=2, J_{c}(3,2)=5+3 z$. The elements of $S_{3} \times S_{4}$ whose second permutation has one big number are $(12)(3),(12)(3)(4) ;(12)(3),(13)(2)(4)$; $(13)(2),(12)(3)(4) ; \quad(13)(2),(13)(2)(4) ; \quad(1)(23),(1)(23)(4) ;$ the other 3 elements are (1)(23), (1)(24)(3); (12)(3), (14)(2)(3); (13)(2), (14)(2)(3).

\subsection{Another interpretation of $h_{n}(f(1), \ldots, f(k))$}

In the previous subsection we have a combinatorial interpretation of the Jacobi-Stirling numbers of the first kind, but not of the second kind. In this subsection we want to use an idea similar to the ordered cycle structure to obtain another combinatorial interpretation for the Jacobi-Stirling numbers of the second kind and, more generally, of $h_{n}(f(1), \ldots, f(k))$, with $f$ as usual.

Let we denote with $\bar{S}_{n}^{h}$ be the set of ordered sequences of $h$ elements, where the $i$-th element is an ordered finite sequence (maybe empty) of integers in $\{i, i+1, \ldots, n\}$. Given an element $s \in \bar{S}_{n}^{h}$ we say that $s$ has dimension $k$ if the sum of the cardinality of all the $h$ sequences is $k$. For example, in $\bar{S}_{4}^{3}(3,1,4),(4,4,3,4),()$ is an element of dimension 7 .

As done for the permutations, we say that two elements in $\bar{S}_{m}^{h}$ and $\bar{S}_{n}^{h}$ have the same ordered structure if the cardinalities of the sequences of both elements are equal. For example $(3,1,2),(4,4),(3)$ and $(1,2,1),(3,3),(3)$ have the same ordered structure.

Proposition 21. Let $r \in \mathbb{P}, a_{1}, \ldots, a_{r} \in \mathbb{N}$ and $f(x)=\left(x+a_{1}\right) \cdots\left(x+a_{r}\right)$. Then for all $n, k \in \mathbb{N} h_{n}(f(1), \ldots, f(k))$ is the number of elements in $\bar{S}_{k+a_{1}}^{k} \times \cdots \times \bar{S}_{k+a_{r}}^{k}$ with the same ordered structure and dimension $n$.

Proof. If $n=0$ then we have only the empty sequence, indeed $h_{0}(f(1), \ldots, f(k))=1$. If $k=0$ then $\bar{S}_{n}^{0}$ has the trivial element only if $n=0$. Indeed, $h_{n}(0)=0$ for $n \geq 1$. Now let $n \geq 1$ and suppose that the thesis is true for smaller values of $n$. Fix an element $s=\left(s_{i}\right)_{i \leq k} \in \bar{S}_{k+a_{1}}^{k} \times \cdots \times \bar{S}_{k+a_{r}}^{k}$. For all $i \leq k$ the cardinality of the first sequence in $s_{i}$ is the same for all $i$ and it ranges between 0 and $n$ : we call $c$ this number. Now each number in the first sequence in $s_{i}$ is in $\left\{1, \ldots, k+a_{i}\right\}$. We delete the first sequence in each $s_{i}$ and we decrease all numbers by one. We obtain elements in $\bar{S}_{k-1+a_{1}}^{k-1} \times \cdots \times \bar{S}_{k-1+a_{r}}^{k-1}$ with dimension $n-c$. By induction there are $h_{n-c}(f(1), \ldots, f(k-1))$ of such elements. In formula, the cardinality of $\bar{S}_{k+a_{1}} \times \cdots \times \bar{S}_{k+a_{r}}$ is

$$
\begin{aligned}
\sum_{c=0}^{n} h_{n-c}(f(1), \ldots, f(k-1)) \prod_{i=1}^{r}\left(k+a_{i}\right)^{c} & =\sum_{c=0}^{n} h_{n-c}(f(1), \ldots, f(k-1)) f(k)^{c} \\
& =h_{n}(f(1), \ldots, f(k)) .
\end{aligned}
$$

Thus the proof is completed.

By (18) we have

Corollary 22. Let $n, j, z \in \mathbb{N}$. Then $J S(n, j)$ is the number of elements in $\bar{S}_{j}^{j} \times \bar{S}_{j+z}^{j}$ with the same ordered structure and dimension $n-j$. 
We now give an example when $z=1$. We know that $L S(3,2)=8$. The elements in $\bar{S}_{2}^{2} \times \bar{S}_{3}^{2}$ as in Corollary 22 are the following: (1)(), (1)(); (1)(), (2)(); (1)(), (3)(); (2)(), (1)(); $(2)(),(2)() ;(2)(),(3)() ;()(2),()(2) ;()(2),()(3)$.

If we consider $h_{n}(f(1), \ldots, f(k))$ as polynomial in $a_{1}, \ldots, a_{r}$ we have the following result.

Proposition 23. Let $r \in \mathbb{P}, \beta_{1}, \ldots, \beta_{r} \in \mathbb{N}$ and $f(x)=\left(x+a_{1}\right) \cdots\left(x+a_{r}\right)$. Then for all $n, k \in \mathbb{N}$ the coefficient of $a_{1}^{\beta_{1}} \cdots a_{r}^{\beta_{r}}$ in $h_{n}(f(1), \ldots, f(k))$ is the number of elements $s=\left(s_{i}\right)_{i \leq r} \in\left(\bar{S}_{k+1}^{k}\right)^{r}$ with the same ordered structure, dimension $n$ and such that $k+1$ appears $\beta_{i}$ times in all sequences of $s_{i}$ for all $i \leq r$.

The proof is essentially the same as that of Proposition 21. In the case of the JacobiStirling numbers of the second kind, this proposition becomes

Corollary 24. Let $n, j, b \in \mathbb{N}$. Then the coefficient of $z^{b}$ in $J S_{n}^{(j)}(z)$ is the number of elements in $\bar{S}_{j} \times \bar{S}_{j+1}$ with the same ordered structure and dimension $n-j$, such that $j+1$ appears $b$ times.

\subsection{Monomial symmetric functions}

In this last subsection we want to give a simple combinatorial interpretation of the monomial symmetric functions, evaluated in $f(1), \ldots, f(k)$, where $f$ is as usual.

Let $\lambda=\left(\lambda_{1}, \ldots, \lambda_{t}\right)$ be a partition of $n=|\lambda|$ and let $k$ be an integer, $k \geq t$. Then the monomial symmetric function associated to $\lambda$ in $x_{1}, \ldots, x_{k}$ is

$$
m_{\lambda}\left(x_{1}, \ldots, x_{k}\right)=\sum_{\sigma} x_{\sigma(1)}^{\lambda_{1}} \cdots x_{\sigma(k)}^{\lambda_{k}}
$$

where the sum is over the group of permutations $S_{k}$ modulo the stabilizer of $\lambda$. We will give a combinatorial interpretation of $m_{\lambda}(f(1), \ldots, f(k))$. Let $\mathbf{u}=\left(u_{1}, \ldots, u_{k}\right), \mathbf{v}=$ $\left(v_{1}, \ldots, v_{k}\right) \in \mathbb{N}^{k}$ be two sequences of $k$ integers. We say that $\mathbf{u} \geq \mathbf{v}$ if $u_{i} \geq v_{i}$ for all $i \leq k$. Moreover, if $m \in \mathbb{N}$ we say that $\mathbf{u} \leq m$ if $u_{i} \leq m$ for all $i \leq k$.

Fix a positive integer $k$ and a partition $\lambda=\left(\lambda_{1}, \ldots, \lambda_{t}\right)$, with $k \geq t$. Let $n=|\lambda|$. We define $S_{\lambda, k}$ as the set of $n$-tuples of elements $\leq k$ in increasing order such that $\{m(1), m(2), \ldots, m(k)\}=\left\{\lambda_{1}, \ldots, \lambda_{k}\right\}$ as multiset, where $m(i)$ denotes the multiplicity of $i$ and $\lambda_{i}=0$ for all $i>t$. For example, if $k=3, \lambda=(2,1)$ then $S_{\lambda, k}=\{(1,1,2),(1,1,3),(1,2,2),(1,3,3),(2,2,3),(2,3,3)\}$.

Proposition 25. Let $r \in \mathbb{P}, a_{1}, \ldots, a_{r} \in \mathbb{N}$ and $f(x)=\left(x+a_{1}\right) \cdots\left(x+a_{r}\right)$. Then $m_{\lambda}(f(1), \ldots, f(k))$ is the number of $r+1$ sequences $\left(s_{0}, \ldots, s_{r}\right)$, not necessarily ordered, each one with $n=|\lambda|$ elements, such that $s_{0} \in S_{\lambda, k}$ and $s_{0} \leq s_{i} \leq k+a_{i}$ for all $i \leq k$.

Proof. Let $s_{0}=\left(j_{1}, \ldots, j_{n}\right) \in S_{\lambda, k}$. Then for all $i \leq r$ there are $\left(k+a_{i}-j_{1}+1\right)\left(k+a_{i}-\right.$ $\left.j_{2}+1\right) \cdots\left(k+a_{i}-j_{n}+1\right)$ sequences $s_{i}$ such that $s_{0} \leq s_{i} \leq k+a_{i}$. Therefore there are

$$
\prod_{i=1}^{r}\left(\prod_{h=1}^{n}\left(k+a_{i}-j_{h}+1\right)\right)=\prod_{h=1}^{n} f\left(k-j_{h}+1\right)=\prod_{j=1}^{k} f(j)^{m\left(k+1-j, s_{0}\right)}
$$


sequences $\left(s_{0}, \ldots, s_{r}\right)$ as in the statement, with $s_{0}$ fixed $\left(m\left(j, s_{0}\right)\right.$ denotes the multiplicity of $j$ in $s_{0}$ ). Now we sum over $s_{0}$ and by virtue of definition of $S_{\lambda, k}$ we have the number of all such sequences is

$$
\sum_{s_{0} \in S_{\lambda, k}} \prod_{j=1}^{k} f(j)^{m\left(k+1-j, s_{0}\right)}=m_{\lambda}(f(1), \ldots, f(k)) .
$$

When $\lambda=(1,1, \ldots, 1)$ we have that $m_{\lambda}\left(x_{1}, \ldots, x_{r}\right)=e_{|\lambda|}\left(x_{1}, \ldots, x_{r}\right)$. Therefore we have the following result.

Corollary 26. Let $n, j, z \in \mathbb{N}$. Then $J c_{n}^{(j)}(z)$ is the number of sequences $\left(s_{0}, s_{1}, s_{2}\right)$, each one with $n-j$ elements, such that the elements of $s_{0}$ are strictly increasing and $s_{0} \leq s_{1} \leq k$ and $s_{0} \leq s_{2} \leq n-1+z$ (the sequences $s_{1}$ and $s_{2}$ are not necessarily ordered).

For example, let $n=3, j=1, z=1$. Then $L c_{3}^{(1)}=12$. Here we list all 12 sequences satysfying the previous statement: $\{(1,2),(1,2),(1,2)\},\{(1,2),(1,2),(1,3)\}$, $\{(1,2),(1,2),(2,2)\}, \quad\{(1,2),(1,2),(2,3)\}, \quad\{(1,2),(1,2),(3,2)\}, \quad\{(1,2),(1,2),(3,3)\}$, $\{(1,2),(2,2),(1,2)\}, \quad\{(1,2),(2,2),(1,3)\}, \quad\{(1,2),(2,2),(2,2)\}, \quad\{(1,2),(2,2),(2,3)\}$, $\{(1,2),(2,2),(3,2)\},\{(1,2),(2,2),(3,3)\}$.

If we want to consider $m_{\lambda}(f(1), \ldots, f(k))$ as polynomial in $a_{1}, \ldots, a_{r}$ then we have the following interpretation.

Proposition 27. Let $r \in \mathbb{P}, \beta_{1}, \ldots, \beta_{r} \in \mathbb{N}$ and $f(x)=\left(x+a_{1}\right) \cdots\left(x+a_{r}\right)$. Then the coefficient of $a_{1}^{\beta_{1}} \cdots a_{r}^{\beta_{r}}$ in $m_{\lambda}(f(1), \ldots, f(k))$ is the number of $r+1$ sequences $\left(s_{0}, \ldots, s_{r}\right)$, not necessarily ordered, each one with $n=|\lambda|$ elements, such that $s_{0} \in S_{\lambda, k}$ and $s_{0} \leq s_{i} \leq$ $k+1$ for all $i \leq k$, such that $k+1$ appears $\beta_{i}$ times in $s_{i}$.

Corollary 28. Let $n, j, b \in \mathbb{N}$. Then the coefficient of $z^{b}$ in $J c_{n}^{(j)}(z)$ is the number of sequences $\left(s_{0}, s_{1}, s_{2}\right)$, not necessarily ordered, each one with $n=|\lambda|$ elements, such that $s_{0}$ has its elements pairwise distinct and $s_{0} \leq s_{1} \leq k$ and $s_{0} \leq s_{2} \leq k+1$, such that $k+1$ appears $b$ times.

\section{$6 \quad$ Final remarks}

In this section we recall properties of symmetric functions and apply them to the special symmetric functions $H_{j, n}^{f}$ and $E_{j, n}^{f}$. It is well known (see e.g. [12, Chapter I.2]) that the generating functions of the elementary and complete symmetric functions are respectively

$$
\begin{aligned}
\sum_{r=0}^{n} e_{r}\left(x_{1}, \ldots, x_{n}\right) t^{r} & =\prod_{i=1}^{n}\left(1+x_{i} t\right) \\
\sum_{r=0}^{\infty} h_{r}\left(x_{1}, \ldots, x_{n}\right) t^{r} & =\prod_{i=1}^{n} \frac{1}{1-x_{i} t}
\end{aligned}
$$


Therefore, the generating functions of $E_{j, n}^{f}$ and $H_{j, n}^{f}$ are respectively

$$
\begin{aligned}
\sum_{j=0}^{n} E_{j, n}^{f} t^{n-j} & =\prod_{i=1}^{n-1}(1+f(i) t) \\
\sum_{n=j}^{\infty} H_{j, n}^{f} t^{n-j} & =\prod_{i=1}^{j} \frac{1}{1-f(i) t}
\end{aligned}
$$

or, equivalently,

$$
\begin{aligned}
& \sum_{j=0}^{n} E_{j, n}^{f} t^{j}=t \prod_{i=1}^{n-1}(t+f(i)) \\
& \sum_{n=j}^{\infty} H_{j, n}^{f} t^{n}=\prod_{i=1}^{j} \frac{t}{1-f(i) t} .
\end{aligned}
$$

We have that the matrices $H^{f}=\left(H_{j, n}^{f}\right)_{j, n \in \mathbb{N}}$ and $E^{f}=\left((-1)^{j+n} E_{j, n}^{f}\right)_{j, n \in \mathbb{N}}$ are inverses of each other. In fact, for fixed $j, n^{\prime} \in \mathbb{N}$

$$
\begin{aligned}
\frac{\prod_{i=1}^{n^{\prime}-1}(1-f(i) t)}{\prod_{l=1}^{j}(1-f(l) t)} & =\left(\sum_{n=0}^{\infty} H_{j, n}^{f} t^{n-j}\right)\left(\sum_{j^{\prime}=0}^{n^{\prime}} E_{j^{\prime}, n^{\prime}}^{f}(-t)^{n^{\prime}-j^{\prime}}\right) \\
& =\sum_{n=0}^{\infty} \sum_{j^{\prime}=0}^{n^{\prime}}(-1)^{j^{\prime}+n^{\prime}} H_{j, n}^{f} E_{j^{\prime}, n^{\prime}}^{f} t^{n-j+n^{\prime}-j^{\prime}} .
\end{aligned}
$$

When we extract the coefficient of $t^{n^{\prime}-j}$, by (31), we get the entry $j, n^{\prime}$ of the product $H^{f} E^{f}$. If $n^{\prime}<j$ then $t^{n^{\prime}-j}$ has coefficient 0 in the RHS of (30); if $n^{\prime}=j$ the LHS of (30) is $\frac{1}{1-f(j) t}$ and therefore the coefficient of $t^{0}$ is 1 ; if $n^{\prime}>j$ the LHS of (30) is a polynomial of degree $n^{\prime}-j-1$ and therefore the coefficient of $t^{n^{\prime}-j}$ is 0 . So the product $H^{f} E^{f}$ is the (infinite) identity matrix.

Now, for all $j \geq 1$, set $\langle x\rangle_{j}:=x(x-f(1))(x-f(2)) \cdots(x-f(j-1))$ and set $\langle x\rangle_{0}:=1$. By previous remark and by (28) we have

$$
\begin{aligned}
x^{n} & =\sum_{j=0}^{n} H_{j, n}^{f}\langle x\rangle_{j} \\
\langle x\rangle_{n} & =\sum_{j=0}^{n} E_{j, n}^{f} x^{j} .
\end{aligned}
$$

Finally, since $f$ has all real and nonpositive zeros, $f$ is injective if it is evaluated on $\mathbb{R}^{+}$. Thus, it is possible to use the Newton interpolation formula

$$
x^{n}=\sum_{j=0}^{n}\left(\sum_{r=0}^{j} \frac{x_{r}^{n}}{\prod_{k=0, k \neq r}^{j}\left(x_{r}-x_{k}\right)}\right) \prod_{i=0}^{j-1}\left(x-x_{i}\right)
$$


in (32) to obtain the following expression for the $H_{j, n}^{f}$ when $n \geq 1$ (we set $x_{0}=0$ )

$$
H_{j, n}^{f}=\sum_{r=1}^{j} \frac{f(r)^{n-1}}{\prod_{k=1, k \neq r}^{j}(f(r)-f(k))} .
$$

\section{Acknowledgements}

I wish to thank my advisor Prof. Francesco Brenti for his advice, support, and constant encouragement during the course of my research.

\section{References}

[1] G. E. Adrews, W. Gawronski, L. L. Littlejohn, The Legendre-Stirling numbers Discrete Math., 311:1255-1272, 2011.

[2] G. E. Andrews, L. L. Littlejohn, A combinatorial interpretation of the LegendreStirling numbers. Proc. AMS, 137(8):2581-2590, 2009.

[3] F. Brenti, Combinatorics and Total Positivity J. Combin. Th., Ser. A, 71(2):175-218, 1995.

[4] L. Carlitz, On abelian fields Trans. Amer. Math. Soc., 35:122-136, 1933.

[5] E. S. Egge, Legendre-Stirling permutations Europ. J. of Comb. 31(7):1735-1750, 2010.

[6] W. N. Everitt, L. L. Littlejohn, R. Wellman, Legendre polynomials, Legendre-Stirling numbers, and the left-definite analysis of the Legendre differential expression. $J$. Comput. Appl. Math. 148(1):213-238, 2002.

[7] W. N. Everitt, K. H. Know, L. L. Littlejohn, R. Wellman, G. J. Yoon, JacobiStirling numbers, Jacobi polynomials, and the left-definite analysis of the classical Jacobi differential expression. J. Comput. Appl. Math. 208(1):29-56, 2007.

[8] Y. Gelineau, J. Zeng, Combinatorial interpretations of the Jacobi-Stirling numbers. Electron. J. Combin. 17, \#R70, 2010.

[9] I. Gessel, G. Viennot, Determinants, Paths and Plane Partitions. preprint, 1989.

[10] H. W. Gould, The $q$-Stirling numbers of the first and second kinds. Duke Math. J. 28:281-289, 1961.

[11] P. Leroux, Reduced matrices and $q$-log concavity properties of $q$-Stirling numbers. J. Combin. Theory Ser. A 54(1):64-84, 1990.

[12] I. G. Macdonald, Symmetric functions and Hall polynomials Oxford Univ. Press, New York, 1995.

[13] P. Mongelli, Total positivity properties of Jacobi-Stirling numbers. Adv. Appl. Math. 48(2):354-364, 2012.

[14] B. Sagan, A maj Statistic for Set Partitions. Europ. J. Combin. 12:69-79, 1991. 